\title{
The Use of Titanium Mesh Cages in the Reconstruction of Anterior Column Defects in Active Spinal Infections: Can We Rest the Crest?
}

\author{
Gabriel David Sundararaj, Rohit Amritanand, Krishnan Venkatesh, Justin Arockiaraj \\ Spinal Disorders Surgery Unit, Department of Orthopaedics, Christian Medical College, Vellore, Tamil Nadu, India
}

Study Design: Retrospective clinical series.

Purpose: To assess whether titanium cages are an effective alternative to tricortical iliac crest bone graft for anterior column reconstruction in patients with active pyogenic and tuberculous spondylodiscitis.

Overview of Literature: The use of metal cages for anterior column reconstruction in patients with active spinal infections, though described, is not without controversy.

Methods: Seventy patients with either tuberculous or pyogenic vertebral osteomyelitis underwent a single staged anterior debridement, reconstruction of the anterior column with titanium mesh cage and adjuvant posterior instrumentation. The lumbar spine was the predominant level of involvement. Medical co-morbidities were seen in 18 (25.7\%) patients. A significant neurological deficit was seen in $32(45.7 \%)$ patients. At follow up patients were assessed for healing of disease, bony fuson, and clinical outcome was assessed using Macnab's criteria.

Results: Final follow up was done on 64 (91.4\%) patients at a mean average of 25 months (range, 12 to 110 months). Pathologic organisms could be identified in $42(60 \%)$ patients. Forty two (60\%) patients had histopathological findings consistent with tuberculosis. Thirty of 32 (93.7\%) patients showed neurological recovery. The surgical wound healed uneventfully in $67(95.7 \%)$ patients. Bony fusion was seen in $60(93.7 \%)$ patients. At final follow up healing of infection was seen in all patients. As per Macnab's criteria 61 (95.3\%) patients reported a good to excellent outcome.

Conclusions: Inspite of the theoretical risks, titanium cages are a suitable alternative to autologous tricortical iliac crest bone graft in patients with active spinal infections.

Key Words: Thoracolumbar spine, Discitis, Anterior column reconstruction, Titanium cage

\section{Introduction}

Meticulous debridement culminating in the extirpation of all infected tissue is the aim of the surgical management of spinal infections $[1,2]$. This often leads to the creation of substantial anterior column defects which then need to be reconstructed. For decades now, the tricortical iliac crest autologous bone graft has been the gold standard for this surgical exercise [3-5]. It's harvest however is hampered by a number of serious limitations in terms of considerable donor site morbidity [4,5], inadequate availability in lengthy defects [4] and the potential of graft slippage and fracture [6]. Additionally chronic donor site pain has been reported in up to $39 \%$ of patients [5,7]. It therefore behoves surgeons to search for alternatives to this hitherto trusted

Received Feb 4, 2011; 1st Revised Mar 15, 2011; Accepted Apr 8, 2011

Corresponding author: Rohit Amritanand, MS

Spinal Disorders Surgery Unit, Department of Orthopaedics, Christian Medical College,

Vellore, Tamil Nadu 632004, India

Tel: +91-41-6-2282020, Fax: +91-41-6-2232035, E-mail: rohit@ cmcvellore.ac.in 
ally.

Considering that titanium cages have established their superiority in the reconstruction of spinal column defects following tumor and trauma surgery [8], they would appear to be the default choice. However, implantation of metal into the lap of infection is a taboo viewed by most surgeons with trepidation and robust criticism [8]. These views endure due to the fact that this paradigm shift conflicts with age old orthopaedic principles, especially ones that have received the sanction of modern in vitro studies that demonstrate the formation of an antibiotic impregnable biofilm by microorganisms on metal implants [9]. Inspite of this a few surgeons have used these implants in infections with good results $[1,4,6,8,10]$ and therefore this technique warrants validation.

In 1993, we began using titanium mesh cages for the reconstruction of the anterior column in patients with active pyogenic and tuberculous spinal infections $[11,12]$. The purpose of this retrospective clinical study was to investigate the option of using titanium cages as an alternative to the tricortical iliac crest autologous bone graft in a consecutive series of such patients.

\section{Materials and Methods}

Between January 2000 and June 2009, 70 patients diagnosed at this tertiary care hospital with either tuberculous or pyogenic vertebral osteomyelitis underwent, with prior informed consent, a single staged anterior debridement, reconstruction of the anterior column with titanium mesh cage and adjuvant posterior instrumentation. All patients with a minimum follow up of 12 months were included. Institutional Review Board approval was received for this retrospective clinical study.

The diagnosis of spondylodiscitis was suspected on the clinical presentation, anteroposterior and lateral radiographs, magnetic resonance imaging (MRI), elevated white blood cell (WBC) counts, erythrocyte sedimentation rate (ESR) and C-reactive protein (CRP). This was then

Table 1. Level of involvement

\begin{tabular}{lr}
\hline \hline Level & No. \\
\hline Thoracic & 16 \\
Thorocolumbar & 23 \\
Lumbar & 25 \\
Lumbo-sacral & 6 \\
\hline
\end{tabular}

confirmed by microbiological and histopathological study of tissue retrieved during surgery. In 64 (91.4\%) patients the infection was haematogenous and in the remaining 6 $(8.6 \%)$ patients, it occurred post-procedural after microsurgical lumbar discectomies.

The average age of these patients was 45 years (range, 15 to 73 years). There were 45 (64.2\%) male patients. The lumbar spine was the predominant level of involvement (Table 1). Seven (10\%) patients had two to three contiguous levels of infection.

Medical comorbidities were seen in $18(25.7 \%)$ patients. Of these $5(7.1 \%)$ patients had multiple comorbities (Table 2).

Neurologic status was recorded according to Frankel's grade [13]. A significant neurological deficit was seen in 32 patients (Frankel $\mathrm{A}=11$, Frankel $\mathrm{B}=11$, Frankel $\mathrm{C}=10$ ) $(45.7 \%)$. The radiographic analysis included the preoperative, postoperative, and follow-up measurement of sagittal profile, evaluation of fusion and the assessment of hardware status. The sagittal profile was measured using Konstam's angle [14] which is the angle between the endplates above and below the infected vertebrae. This was measured using Centricity 1.0 (RadiologyWeb, General Electric, Mount Prospect, Milwaukee, WI, USA). MRI was used in all patients to assess the extent of abscess (epidural, pre- or paravertebral) and changes such as oedema and myelomalacia in the spinal cord. Osseous fusion was said to have been achieved if one or more of these parameters was met 1) bony continuity was seen between the diseased vertebra, 2) the presence of a 'sentinel sign' [15], 3) absence of angular movement of the cage on dynamic lateral flexion-extension X-rays. Computed tomograms were also used in $10(14.3 \%)$ patients to study the fusion (Fig. 1E).

Indications for surgery included neurological deficit

Table 2. Comorbidities

\begin{tabular}{lr}
\hline \hline Comorbidity & No. \\
\hline Diabetes & 6 \\
Hypothyroidism & 7 \\
Chronic renal disease & 2 \\
HIV infection & 2 \\
Hypertension & 2 \\
Acute lymphoblastic leukaemia & 1 \\
Osteosarcoma & 1 \\
Ankylosing spondylitis & 1 \\
Intravenous drug abuser & 1 \\
Hepatitis C & 1 \\
\hline
\end{tabular}

HIV: Human immunodeficiency virus. 

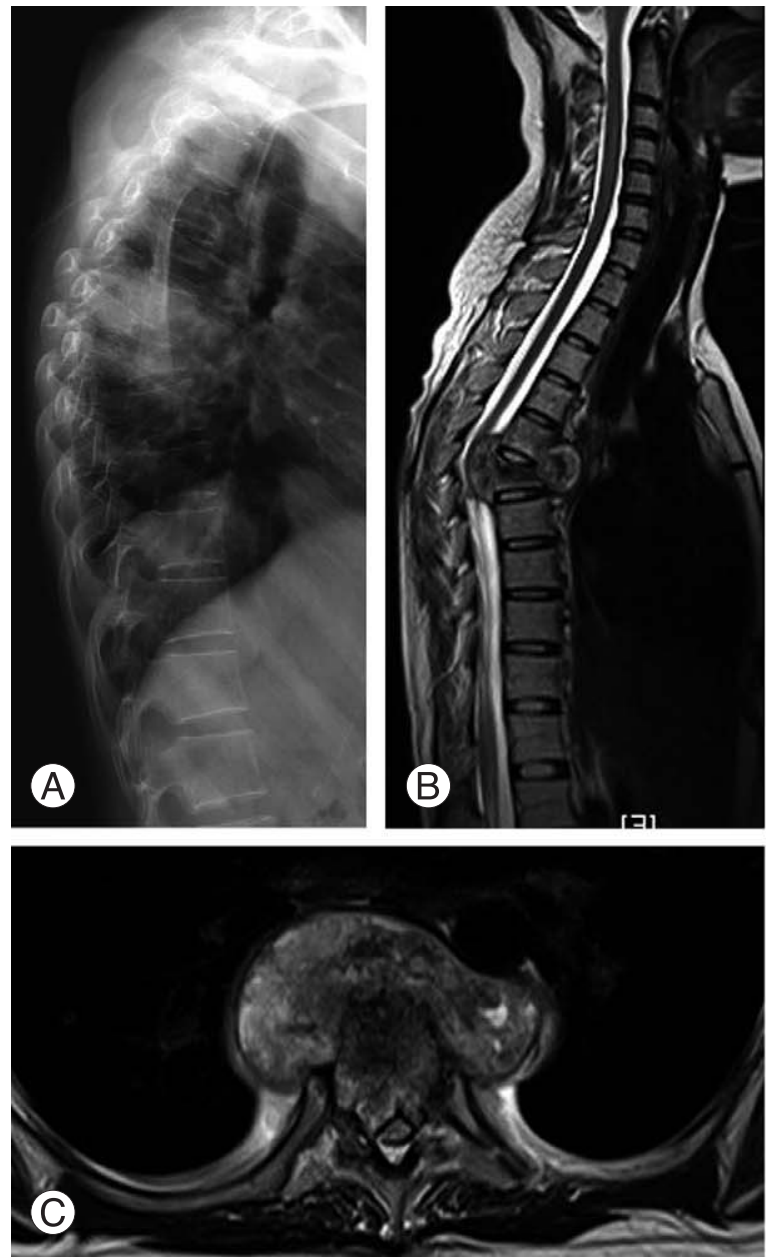


Fig. 1. A 26-year-old female with tuberculous spondylodiscitis at T56 with muti-drug resistant Mycobacterium tuberculosis. (A) Preoperative plain radiograph (lateral view) demonstrating the kyphotic deformity $\left(34^{\circ}\right)$ and partial destruction of T6. (B) Preoperative T2 weighted magnetic resonance imaging (MRI), sagittal section demonstrating the epidural and anterior vertebral abscess. (C) Preoperative T2 weighted MRI, axial section showing the large anterior abscess. (D) Follow up plain radiograph (lateral view) at 21 months showing fusion and the intact implants. (E) Computed tomogram (sagittal section) facilitating the demonstration of interbody fusion at T5-6.
(Frankel A, Frankel B, Frankel C), severe unrelenting pain which prevented a patient from standing or sitting without using their hands for support, substantial vertebral body destruction leading to local instability or failure of nonoperative management [12] .

In $58(82.8 \%)$ patients a 'posterior-only' approach [16] was used to place pedicle screws, carry out the anterior column debridement and seat the cage (Fig. 1). In the early period of this study, prior to familiarity with the 'posterioronly' approach, $9(12.8 \%)$ patients had a separate approach to the anterior column while in $3(4.2 \%)$ patients, the cage was deemed too long to be safely placed through a posterior approach. Irrespective of the approach, drainage of pus and a meticulous debridement of all infected granulation tissue, devitalised disc and sequestra were carried out, followed by a thorough curettage to bleeding cancellous bone. Decompression of the spinal canal was undertaken when necessary. The surgical defect thus created was carefully measured, a titanium mesh cage of appropriate diameter and length was cut and filled with morcellised bone graft, tapped into the defect and the remaining space was packed with autologous bone. Bone graft was obtained from the resected ribs in thoracic level disease or from the uninfected posterior elements in lumbar level disease. Mesh cages were placed intersomatically in 57 patients, replaced one vertebra in 10 patients, two vertebrae in 1 patient, and three vertebral bodies in 2 patients. Intra-operative specimens were sent for Gram and acid-fast Bacillus staining, culture and sensitivity for pyogenic and tuberculous organisms, and histological examination. Polyaxial pedicle screws were used in 66 (94.2\%) patient. A Hartshill rectangle (Surgicraft, Redditch, UK) [17] and sublaminar wires was used in 3 patients and Steffee plates (Acromed, Cleveland, $\mathrm{OH}$, USA) [18] and screws in 1 patient. Depending on the neurological status, patients were either ambulated or wheelchair mobilised within the first 2-3 days after surgery. All patients were treated with appropriate culture specific antibiotics. In patients with pyogenic organism the antibiotics were given intravenously for the first two weeks and then orally for 10-12 weeks [12]. In the early part of this 
study (2000-2005) those infected with Mycobacterium tuberculosis received antituberculous therapy for 18 months, after 2006 this duration was reduced to 9 months as suggested by the infectious disease specialists [19].

All patients were followed up at 3 months, 6 months, 12 months and then yearly. At each follow up patients were assessed for residual pain, resolution of infection, neurological grade, kyphos angle and the state of the fusion and implant. Clinical outcome was assessed using Macnab's criteria [20]. Disease was deemed to have healed if there was an absence of pain, roentgenograms revealed bony fusion, ESR and CRP had normalised, and the patients reported a sense of well being.

\section{Results}

Four patients (5.7\%) had inadequate follow up and have been excluded. There were 2 deaths. The first was an emaciated 69-year-old male admitted with D7-8 tuberculous spondylodiscitis, Frankel A paraplegia and bilateral sacral and left scapular pressure sores. In the post operative period he was admitted into the Intensive Care Unit for monitoring where he developed aspiration pneumonia. He recovered from this by the 5th post-operative day with antibiotics and chest physiotherapy, but expired on the 8th post-operative day after a sudden drop in urine output and dyselectrolytemia. The other was a 62-year-old male with D5-6 tuberculous spondylodiscitis and Frankel A paraplegia, who died 4 months after surgery due to unrelated causes. The

Table 3. Microbiological result

\begin{tabular}{lr}
\hline \hline Organism & No. \\
\hline Mycobacterium tuberculosis & 30 \\
Methicillin sensitive Staphylococcus aureus (MSSA) & 4 \\
Methicillin resistant Staphylococcus aureus (MRSA) & 1 \\
Escherichia coli & 1 \\
Pseudomonas areuginosa & 3 \\
Enterobacter & 2 \\
Coagulase negative Staphylococcus aureus & 1 \\
No growth & 28 \\
\hline
\end{tabular}

remaining 64 patients were evaluated at an average mean follow up of 25 months (range, 12 to 110 months).

Pathologic organisms from intraoperative specimen could be identified in $42(60 \%)$ patients (Table 3). Of the 30 (42.8\%) patients with M. tuberculosis the sensitivity demonstrated the multidrug resistant variety in 4 patients. All patients had evidence of osteomyelitis on histopathological examination. Of these 42 (60\%) patients had pathological findings consistent with tuberculosis.

Average preoperative WBC count was $8,786 / \mathrm{mm}^{3}$ (range, 4,800 to $11,000 / \mathrm{mm}^{3}$; normal range, 4,300 to $10,800 / \mathrm{mm}^{3}$ ), ESR was $63.7 \mathrm{~mm} / \mathrm{hr}$ (range, 3 to $136 \mathrm{~mm} / \mathrm{hr}$; normal range, 6 to $12 \mathrm{~mm} / \mathrm{hr}$ ) and CRP was $31.0 \mathrm{mg} / \mathrm{l}$ (normal $<6 \mathrm{mg} / \mathrm{l}$ ). These improved to $7,364.5 / \mathrm{mm}^{3}, 36 \mathrm{~mm} / \mathrm{hr}, 9.42 \mathrm{mg} / \mathrm{l}$, respectively, at final follow up.

Of the $32(45.7 \%)$ patients who showed significant (Frankel A, B, C) preoperative neurologic deficits, one had deterioration of neurological status after surgery following a Brown-Sequard syndrome, however this recovered in 10 days. At final follow up two patients with Frankel A paraplegia showed no improvement, all other patients showed recovery (Table 4).

The surgical wound healed uneventfully in 67 (95.7\%) patients. Three (4.3\%) patients with M. tuberculosis infection required wound lavage and debridement in the early post operative period. Neither grew a new organism from the second procedure. One patient with pyogenic infection had a persistent discharging sinus which healed only when the implant was removed at 12 months after the index surgery. At final follow up healing of infection was seen in all patients.

Table 4. Neurological status

\begin{tabular}{ccc}
\hline \hline Frankel score & Pre-operative & Follow up \\
\hline A & 11 & 2 \\
B & 11 & 0 \\
C & 10 & 2 \\
D & 8 & 2 \\
E & 30 & 58 \\
Total & 70 & 64 \\
\hline
\end{tabular}

Table 5. Mean (range) kyphos angle (as per Konstam, ${ }^{\circ}$ )

\begin{tabular}{lcccc}
\hline \hline Level & Preoperative & Immediate post operative & Final follow up & Loss of correction \\
\hline Thoracic & $23.8(0$ to 95$)$ & $8.1(-10$ to 43$)$ & $12.5(-1$ to 45$)$ & $4.5(0$ to 22$)$ \\
Lumbar & $-10.4(-40$ to 15$)$ & $-17.3(-40$ to 10$)$ & $-15(-38$ to 10$)$ & $2.1(0$ to 12$)$ \\
\hline
\end{tabular}


Bony fusion was seen in 60 (93.7\%) of 64 followed up patients. Of the $4(6.3 \%)$ patients with pseudarthrosis, one patient had subsidence of the titanium cage and a $22^{\circ}$ loss of correction after 34 months with a proximal screw backing out and a prominence under the skin. He was unwilling to undergo any further surgery [12]. The radiological nonunion has been asymptomatic for the remaining 3 patients and none of them have required an additional surgical procedure. The kyphos angle at final follow up and the loss of correction is shown in (Table 5).

In addition to the one patient with pseudarthrosis following a screw back out, hardware complications in the form of Hartshill rectangle breakage, titanium rod breakage and screw failure occurred in one patient each. All occurred after fusion and did not adversely affect the outcome. An iliac vein injury occurred in one patient and accidental durotomy in 3 patients.

Fifty eight $(90.6 \%)$ patients reported complete resolution of pain and $6(9.4 \%)$ had mild residual pain which did not interfere with their daily activities or require regular analgesics. As per Macnab's criteria, 61 (95.3\%) patients reported a good to excellent outcome and 3 a fair outcome. None had a poor outcome and there were no late recurrences of infection.

\section{Discussion}

The most serious argument against the use of titanium cages arises from the belief that all foreign materials (including metallic implants and allograft) [21] tend to act as a readymade substrates for bacterial adherence and biofilm formation thus reducing antibiotic penetration [9]. In vitro studies suggest that because antibiotics, phagocytes and other humoral immune responders cannot reach bacteria through biofilm, these infections are relatively resistant to host defence mechanisms and antibiotics $[2,6]$. There are however, a number of variables in terms of bacterial species, bacterial load and the characteristics and composition of the metal in question. Therefore, M. tuberculosis shows less adherence than Staphylococcus, and titanium alloys (the predominant metal used in this series) are less prone to colonisation than stainless steel [4]. The use of posterior instrumentation in spinal infection may still be justified because the implant traverses relatively healthy tissue, whereas the titanium cage is seated into the lap of infection.

The outcomes of this clinical study reveal a high rate of healing, adequate restoration and maintenance of sagittal alignment, early pain free mobilisation, a low rate of complications and no late recurrences of infection. We would agree with Christodoulou et al. [6] who believe that the use of cages provides a more secure, accurate and dependable deformity correction than when structural bone graft is used. The loss of correction in our study was only $3^{\circ}$. This is similar to the figures of $3.9^{\circ}$ and $2^{\circ}$ reported by other authors [8,10]. Talu et al. [22] reported graft slippage in 3 and resorption in 2 of 127 patients. These complications are easily overcome with the use of cages, as they have teeth which 'bite' into the adjacent vertebra when compressed, facilitating a secure purchase within bone. This has the bonus of being available in the immediate post operative period, permitting the patient ambulation while at the same time allowing the diseased parts to 'rest.'

It is also pertinent to note that we do not have a separate surgical strategy for patients with tuberculous and pyogenic spondylodiscitis and neither for patients who are immunocompromised $(\mathrm{n}=13,20.3 \%)$ or drug resistant $(\mathrm{n}=5,7 \%)$ (Fig. 1). Once the decision to surgically intervene is made, the focus is on a thorough anterior column debridement, retrieval of adequate tissue for microbiological and histopathological study, a stable reconstruction of the anterior column and finally supplementation with posterior instrumentation. This reinforces the fact that if the debridement is meticulous and the appropriate antibiotic is administered adequately, the in vivo response is the same for the range of microbiological organisms likely to cause spondylodiscitis.

Still others [4] have suggested staging surgery on patients with spinal infections who require the implantation of metal instrumentation. The suggested benefits are that the infected focus is debrided first, thereby reducing the bacterial load before the application of metal. This also provides the patients an interim period during which their nutritional status may be boosted. We do not subscribe to this philosophy and believe that patients are physiologically at their best on the days prior to the first surgery and the addition of another surgical procedure within weeks of the first not only prolongs the hospitalisation, adding to cost, but also perhaps is a double surgical burden to the patients already compromised system [10]. We have always carried out the surgery in a single stage, with gratifying results [12].

So how does one explain this apparent dichotomy from standard orthopaedic teaching? Perhaps the vascular cancellous vertebral bone that metal cages are seated within is 
more forgiving than hard cortical bone, perhaps it is the reconstruction of the erstwhile unstable skeletal elements [23] that augments healing by providing a chance for the in growth of new blood vessels, perhaps it is the rapid pain relief providing the opportunity for immediate ambulation and loading of the spine that promotes bony fusion.

The limitations of this study are similar to those inherent in any retrospective study. Pre- and post-operative objective functional scores were not available preventing us from reporting on the functional benefits of this procedure. We did not compare our results with a cohort of similar patients treated with tricortical crest bone graft. Nevertheless this is one of the largest series of patients with spondylodiscitis managed with this strategy. There was consistency in management because all patients were operated by a single team lead by the senior surgeon and the surgical strategy has essentially remained the same over the years.

\section{Conclusions}

In conclusion, the results of this clinical study reveals that when the fundamental principles of a thorough debridement, restoration of functional stability of the spine and the administration of appropriate antibiotics is adhered to, the use of titanium mesh cage in patients with active spinal infections is safe and the benefits accrued greatly outweigh the potential risks. The titanium mesh cage is a worthy replacement to the iliac crest, a tissue that has served its purpose well and one that has now earned itself a well deserved rest.

\section{REFERENCES}

1. Robinson Y, Tschoeke SK, Kayser R, Boehm H, Heyde CE. Reconstruction of large defects in vertebral osteomyelitis with expandable titanium cages. Int Orthop 2009;33:745-9.

2. Korovessis P, Repantis T, Iliopoulos P, Hadjipavlou A. Beneficial influence of titanium mesh cage on infection healing and spinal reconstruction in hematogenous septic spondylitis: a retrospective analysis of surgical outcome of twenty-five consecutive cases and review of literature. Spine (Phila Pa 1976) 2008;33:E759-67.

3. Hodgson AR, Stock FE, Fang HS, Ong GB. Anterior spinal fusion: the operative approach and pathological findings in 412 patients with Pott's disease of the spine. Br J Surg 1960;48:172-8.
4. Fayazi AH, Ludwig SC, Dabbah M, Bryan Butler R, Gelb DE. Preliminary results of staged anterior debridement and reconstruction using titanium mesh cages in the treatment of thoracolumbar vertebral osteomyelitis. Spine J 2004;4:388-95.

5. Sasso RC, LeHuec JC, Shaffrey C; Spine Interbody Research Group. Iliac crest bone graft donor site pain after anterior lumbar interbody fusion: a prospective patient satisfaction outcome assessment. J Spinal Disord Tech 2005;18 Suppl:S77-81.

6. Christodoulou AG, Givissis P, Karataglis D, Symeonidis PD, Pournaras J. Treatment of tuberculous spondylitis with anterior stabilization and titanium cage. Clin Orthop Relat Res 2006;444:60-5.

7. Silber JS, Anderson DG, Daffner SD, et al. Donor site morbidity after anterior iliac crest bone harvest for single-level anterior cervical discectomy and fusion. Spine (Phila Pa 1976) 2003;28:134-9.

8. Ruf M, Stoltze D, Merk HR, Ames M, Harms J. Treatment of vertebral osteomyelitis by radical debridement and stabilization using titanium mesh cages. Spine (Phila Pa 1976) 2007;32:E275-80.

9. Ha KY, Chung YG, Ryoo SJ. Adherence and biofilm formation of Staphylococcus epidermidis and Mycobacterium tuberculosis on various spinal implants. Spine (Phila Pa 1976) 2005;30:38-43.

10. Kuklo TR, Potter BK, Bell RS, Moquin RR, Rosner MK. Single-stage treatment of pyogenic spinal infection with titanium mesh cages. J Spinal Disord Tech 2006;19:37682.

11. Sundararaj GD, Behera S, Ravi V, Venkatesh K, Cherian VM, Lee V. Role of posterior stabilisation in the management of tuberculosis of the dorsal and lumbar spine. J Bone Joint Surg Br 2003;85:100-6.

12. Sundararaj GD, Babu N, Amritanand R, et al. Treatment of haematogenous pyogenic vertebral osteomyelitis by singlestage anterior debridement, grafting of the defect and posterior instrumentation. J Bone Joint Surg Br 2007;89:12015.

13. Frankel HL, Hancock DO, Hyslop G, et al. The value of postural reduction in the initial management of closed injuries of the spine with paraplegia and tetraplegia. I. Paraplegia 1969;7:179-92.

14. Konstam PG, Blesovsky A. The ambulant treatment of spinal tuberculosis. Br J Surg 1962;50:26-38.

15. McAfee PC. Interbody fusion cages in reconstructive operations on the spine. J Bone Joint Surg Am 1999;81:859-80. 
16. Sundararaj GD, Venkatesh K, Babu PN, Amritanand R. Extended posterior circumferential approach to thoracic and thoracolumbar spine. Oper Orthop Traumatol 2009;21:323-34.

17. Dove J. Internal fixation of the lumbar spine. The Hartshill rectangle. Clin Orthop Relat Res 1986;(203):135-40.

18. Steffee AD, Biscup RS, Sitkowski DJ. Segmental spine plates with pedicle screw fixation: a new internal fixation device for disorders of the lumbar and thoracolumbar spine. Clin Orthop Relat Res 1986;(203):45-53.

19. Upadhyay SS, Saji MJ, Yau AC. Duration of antituberculosis chemotherapy in conjunction with radical surgery in the management of spinal tuberculosis. Spine (Phila Pa 1976) 1996;21:1898-903.

20. Macnab I. Negative disc exploration: an analysis of the causes of nerve-root involvement in sixty-eight patients. J Bone Joint Surg Am 1971;53:891-903.

21. McGuire RA, Eismont FJ. The fate of autogenous bone graft in surgically treated pyogenic vertebral osteomyelitis. J Spinal Disord 1994;7:206-15.

22. Talu U, Gogus A, Ozturk C, Hamzaoglu A, Domanic U. The role of posterior instrumentation and fusion after anterior radical debridement and fusion in the surgical treatment of spinal tuberculosis: experience of 127 cases. J Spinal Disord Tech 2006;19:554-9.

23. Sancineto CF, Barla JD. Treatment of long bone osteomyelitis with a mechanically stable intramedullar antibiotic dispenser: nineteen consecutive cases with a minimum of 12 months follow-up. J Trauma 2008;65:1416-20. 